\title{
Prognostic Stage IV Breast Cancer AJCC v8
}

National Cancer Institute

\section{Source}

National Cancer Institute. Prognostic Stage IV Breast Cancer A/CC v8. NCI Thesaurus.

Code C139587.

Stage IV includes: Any T, Any N, M1, G1-3, HER2 Status: Any, ER Status: Any, PR Status:

Any. G1: Low combined histologic grade (favorable); SBR score of 3-5 points. G2:

Intermediate combined histologic grade (moderately favorable); SBR score: 6-7 points.

G3: High combined histologic grade (unfavorable); SBR score of 8-9 points. M1: Distant metastases detected by clinical and radiographic means and/or histologically proven metastases larger than $0.2 \mathrm{~mm}$. (AJCC 8th ed.) 